\title{
NOTAS NOMENCLATURAIS E TAXONÔMICAS EM MELASTOMATACEAE: COMBINAÇÕES NOVAS EM CAMBESSEDESIA DC. E MARCETIA DC.
}

\author{
Angela Borges Martins ${ }^{1}$
}

Recebido em 14.11.94. Aceito em 22.05.95.

\begin{abstract}
RESUMO - (Notas nomenclaturais e taxonômicas em Melastomataceae: Combinações novas em Cambessedesia DC. e Marcetia DC.) Esta nota trata de algumas alterações nomenclaturais e taxonômicas em Cambessedesia e Marcetia. Cinco novas combinações são propostas. Cambessedesia espora var. ilicifolia é elevada a categoria subespecífica. Pyramia é reduzido a categoria seccional subordinada a Cambessedesia.

Palavras-chave: Melastomataceae, Cambessedesia, Marcetia, combinações novas.

ABSTRACT - (Nomenclatural and taxonomic notes in Melastomaceae: new conbinations in Cambessedesia DC. and Marcetia DC.) The present note deals with some nomenclatural and taxonomic changes in Cambessedesia and Marcetia. Five new combinations are proposed in both genera. Cambessedesia espora var. ilicifolia is elevated to subspecies rank. Pyramia is reduced to a sectional rank under Cambessedesia.
\end{abstract}

Key-words: Melastomataceae, Cambessedesia, Marcetia, new combinations.

\section{Introdução}

A circunscrição inicial do gênero Cambessedesia, conforme delimitado por De Candolle (1828), é bastante diferente da atual, não só por abrigar espécies que foram transferidas para Microlicia Don, mas também por não incluir outras posteriormente descritas. Na última revisão efetuada por Cogniaux (1891) os limites do gênero já se encontram melhor estabelecidos, exceto em relação a Pyramia Cham., considerado distinto de Cambessedesia por apresentar espécies com tricomas estrelados e ovário com 5 ou 6 lóculos. Estudando a morfologia destes dois gêneros e de muitos outros da

\footnotetext{
1 Universidade Estadual de Campinas, Instituto de Biologia, Departamento de Botânica. Caixa Postal 6109 - 13081-970 - Campinas, SP.
} 
família Melastomataceae, Martins (1984) verificou que estas características não são suficientes para a separação de gêneros nesta família, já que ambas são extremamente variáveis. Se as diferenças atribuídas a estes gêneros são inconsistentes, as semelhanças observadas quanto à morfologia dos estames e coloração bicolor das pétalas são inegavelmente conclusivas por ocorrerem apenas em espécies de Cambessedesia e Pyramia. Portanto, o gênero Cambessedesia, segundo Martins (1984), engloba também as espécies até agora reconhecidas sob Pyramia.

O gênero Fritzschia Cham. é muito próximo a Marcetia e se caracteriza por apresentar conectivos projetados ventralmente em aurículas livres. Marcetia bahiana (Ule) A. B. Martins foi originalmente descrita por Ule (1908) como Fritzchia bahiana. Martins (1989) observou que os estames desta espécie tem o conectivo totalmente fundido à base das tecas,sem aurículas ou projeções livres, o que tecnicamente a inclui em Marcetia.

\section{Resultados}

Como consequência dos estudos taxonômicos realizados em Cambessedesia e Marcetia os limites genéricos e alguns limites específicos nestes táxons foram reavaliados e diversas modificações taxonômicas e nomenclaturais foram feitas. As combinações novas e alterações de categoria que se tornaram necessárias são relacionadas abaixo.

Cambessedesia DC. sect. Pyramia (Cham.) A. B. Martins, stat. et sect. nov Pyramia Cham., Linnaea 9:458.1834.

Espécie tipo: Cambessedesia pityrophylla (DC.) A. B. Martins

(Pyramia pityrophylla (DC.) Cham.)

Cambessedesia pityrophylla (DC.) A. B. Martins, comb. nov. Basiônimo: Pyramia pityrophylla (DC.) Cham, Linnaea 9:458.1834; Osbeckia pityrophylla DC., Prodr. 3:139.1828.

Cambessedesia salviaefolia (Cham.) A. B. Martins, comb. nov.

Basiônimo: Pyramia salviaefolia Cham., Linnaea 9:458.1834.

Cambessedesia cambessedesioides (Wurdack) A. B. Martins, comb. nov.

Basiônimo:Pyramia cambessedesioides Wurdack, Phytologia 49 (2):150.1981.

Cambessedesia striatella (Naud.) A. B. Martins, comb. nov.

Basiônimo: Pyramia striatella Naud., Ann. Sci. Nat. Ser. 3:16:60. 1851.

Cambessedesia espora subsp. ilicifolia (DC.) A. B. Martins, stat. nov.

Basiônimo: Cambessedesia ilicifolia (DC.) Triana, Trans. Linn. Soc. London

28:24.1871; Cambessedesia espora var. ilicifolia DC., Prodr. 3: 111.1828. 
Marcetia bahiana (Ule) A. B. Martins, comb. nov.

Basiônimo: Fritzschia bahiana Ule, Bot. jb. 42(2-3):236.1908.

\section{Agradecimentos}

A autora agradece ao Dr. Volker Bittrich pelas sugestões e leitura do manuscrito.

\section{Referências bibliográficas}

Cogniaux, A. 1891. Melastomataceae. In A. \& C. de Candolle (eds), Monographiae Phanerogamarum 7:1-1256.

De Candolle, A. P. 1828. Prodromus. 3:99-202.

Martins, A. B. 1984. Revisão taxonômica do gênero Cambessedesia DC. (Melastomataceae). Dissertação de Mestrado, Instituto de Biologia, UNICAMP.

Martins, A. B. 1989. Revisão taxonômica do gênero Marcetia DC. (Melastomataceae). Tese de Doutorado, Instituto de Biologia, UNICAMP.

Ule, E. 1908. Melastomataceae In Beitrage zur Flora von Bahia. I. Bot. Jb. 42(2/3)232-236. 